\title{
OPPA-COPP Regimen
}

National Cancer Institute

\section{Source}

National Cancer Institute. OPPA-COPP Regimen. NCI Thesaurus. Code C67166.

A regimen consisting of vincristine, prednisone, procarbazine and doxorubicin (OPPA)

followed by cyclophosphamide, vincristine, prednisone and procarbazine (COPP), given in combination with radiation therapy for the treatment of female patients with childhood Hodg kin lymphoma with intermediate-high risk features. 